\title{
Spectroscopy of Red Giants of the Sagittarius Dwarf Galaxy
}

\author{
G. Marconi \\ Osservatorio Astronomico di Roma \\ P. Bonifacio \\ Osservatorio Astronomico di Trieste \\ L. Pasquini \\ European Southern Observatory \\ P. Molaro \\ Osservatorio Astronomico di Trieste
}

\begin{abstract}
The photometric study of the Sagittarius dwarf galaxy by Marconi et al. (1998) has suggested the presence of a spread in metallicity $(-0.7 \leq[\mathrm{Fe} / \mathrm{H}] \leq-1.6)$, which may result from different bursts of star formation. We present the results from a Multi Object Slit spectroscopy program carried out at the NTT with the aim to determine spectroscopic abundances of Sagittarius giants selected from the CCD photometry. From our intermediate resolution $(\mathrm{R} \sim 1500)$ spectra, radial velocities are determined to confirm the membership and metallicities are derived by using spectral synthesis codes for stars down to $V \sim 18$, i.e. below the RGB clump. Out of 57 observed stars, 23 have a radial velocity consistent with Sagittarius membership; here we present results for 8 of these. No star with $[\mathrm{Fe} / \mathrm{H}]<-1.0$ is found, three stars are found to be metal-rich.
\end{abstract}

\section{Introduction}

In a study of the kinematics and abundances of the outer regions of the Galactic Bulge, Ibata, Gilmore \& Irwin $(1994,1995)$ discovered a group of stars with heliocentric radial velocity around $140 \mathrm{~km} \mathrm{~s}^{-1}$ and a low velocity dispersion, which could not be accounted for by any model of the Galactic Bulge. The finding was readily interpreted as a dwarf spheroidal galaxy (dSph) which is a satellite of our own Galaxy. At a distance of about $25 \mathrm{kpc}$ Sagittarius is the nearest external galaxy. It is undergoing tidal disruption and is probably in the process of merging with the Galaxy. This could in fact be one of the many merger events which have contributed to shape the present day Galaxy. The colour-magnitude diagram of Sagittarius suggests a spread in metallicity, 


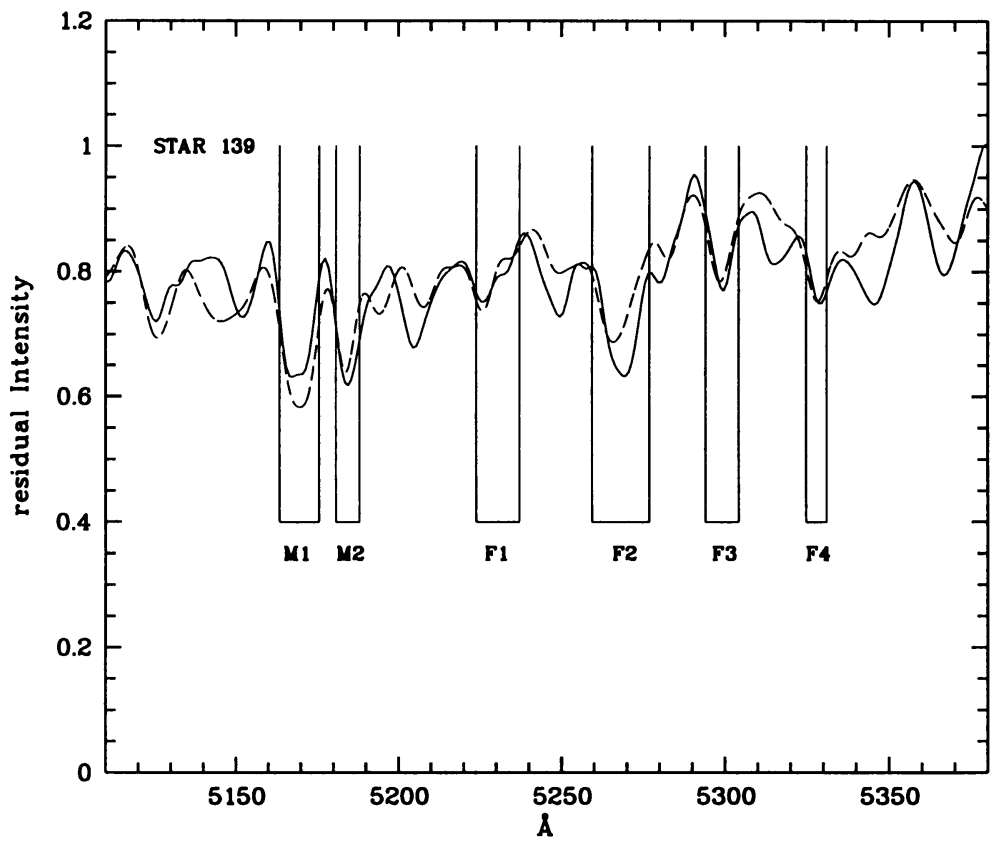

Figure 1. Observed (solid line) and computed spectrum (dashed line) for star 139. The spectra have been smoothed with a Gaussian of width $300 \mathrm{~km} \mathrm{~s}^{-1}$ for display purposes. The solid boxes show the spectrophotometric indices used.

which may result from different bursts of star formation. We have analyzed low resolution spectra NTT+EMMI/MOS to obtain radial velocities and crude estimates of abundances. The method we have developed is quite powerful but the low S/N obtainable with NTT on these $V \approx 18.5$ stars does not allow any firm conclusion on the chemical history of Sagittarius.

\section{Observations}

The observations described here were taken at ESO La Silla on June 19th 1996 with the NTT telescope and EMMI in multi-object-spectroscopy (MOS) mode. We used grism \#5, which provides a resolving power of about $R=\lambda / \Delta \lambda \sim 1500$. The range covered varies slightly depending on the position of the star relative to the center of the field, but the range $480-550 \mathrm{~nm}$ was available for all the stars. The spectra are not useful towards shorter wavelengths due to the falling stellar flux and the decreased efficiency of the CCD. The log of observations is given in Table 1. Details on the observed field can be found in Marconi et al. (1998). 
Table 1. Observations log

\begin{tabular}{cccc}
\hline date & \# of slits & field & $T_{\exp }(\mathrm{s})$ \\
\hline $1998 / 06 / 19$ & 27 & Sgr 1 & 1800 \\
$1998 / 06 / 19$ & 27 & Sgr 1 & 2400 \\
$1998 / 06 / 19$ & 27 & Sgr 1 & 2400 \\
$1998 / 06 / 19$ & 27 & Sgr 1 & 1380 \\
$1998 / 06 / 19$ & 30 & Sgr 1 & 3600 \\
\hline
\end{tabular}

\section{Radial Velocities}

The spectra were calibrated in wavelength using a He-Ar lamp spectrum and zero-point shifts were determined from atmospheric emission lines. To measure radial velocities we used a cross-correlation technique in the $480-530 \mathrm{~nm}$ range. As templates we used synthetic spectra of appropriate temperature and $[\mathrm{Fe} / \mathrm{H}]=$ -0.5 computed with the SYNTHE code (Kurucz 1993). We selected as likely Sgr dSph members all the stars with $100 \leq \mathrm{v}_{\text {hel }} \leq 180 \mathrm{~km} \mathrm{~s}^{-1}$ (Ibata et al. 1997). This left us with a sample of 23 stars out of 57 observed. The velocity dispersion of these stars is $23 \mathrm{~km} \mathrm{~s}^{-1}$. Since the internal velocity dispersion of Sagittarius is only $10 \mathrm{~km} \mathrm{~s}^{-1}$ (Ibata et al. 1995), we assume the dispersion to be dominated by the measurement error of which it may be taken as a somewhat conservative estimate. The radial velocities are given in Table 2, the stars are identified by their number in the Marconi et al. (1998) paper.

\section{Abundances}

For each star we determined the effective temperature from the $(V-I)_{0}$ colour of Marconi et al. (1998) and the calibration of Alonso et al. (1996). Although the calibration is only valid for dwarfs, $V-I$ depends little on gravity; based on theoretical colours computed by Castelli (1998, private communication) we expect to make an error less than $100 \mathrm{~K}$, negligible in this context. Gravities were determined from the isochrones of Straniero, Chieffi \& Limongi (1997) for $8 \mathrm{Gyr}$ and $[\mathrm{Fe} / \mathrm{H}]=-0.5$.

We developed an iterative procedure for the estimate of abundances. For each star we started from a guess of the metallicity and computed a synthetic spectrum. Then both the observed and computed spectra were pseudonormalized using the same pseudo-continuum windows, and the observed spectrum was then normalized by multiplying it by the ratio normalized/pseudonormalized obtained for the synthetic spectrum. On this normalized spectrum we measured four line indices, two of which measure the Mgb triplet (M1 and M2) and four which measure features due to iron-peak elements (F1, F2, F3, F4). Placement of the indices is illustrated in Fig. 1. The indices were defined so as to work efficiently on the sun. Then for each index we iteratively computed its value on the synthetic spectrum, changing the input abundance until it matched the measured index. We first iterated only on the F (iron-peak) indices, at each iteration scaling all of the abundances of the input model. Next we took as scaling the median of the four $\mathrm{F}$ indices and keeping this value fixed we iterated on the $\mathrm{M}$ indices changing only the $\mathrm{Mg}$ abundance. Next we computed a 


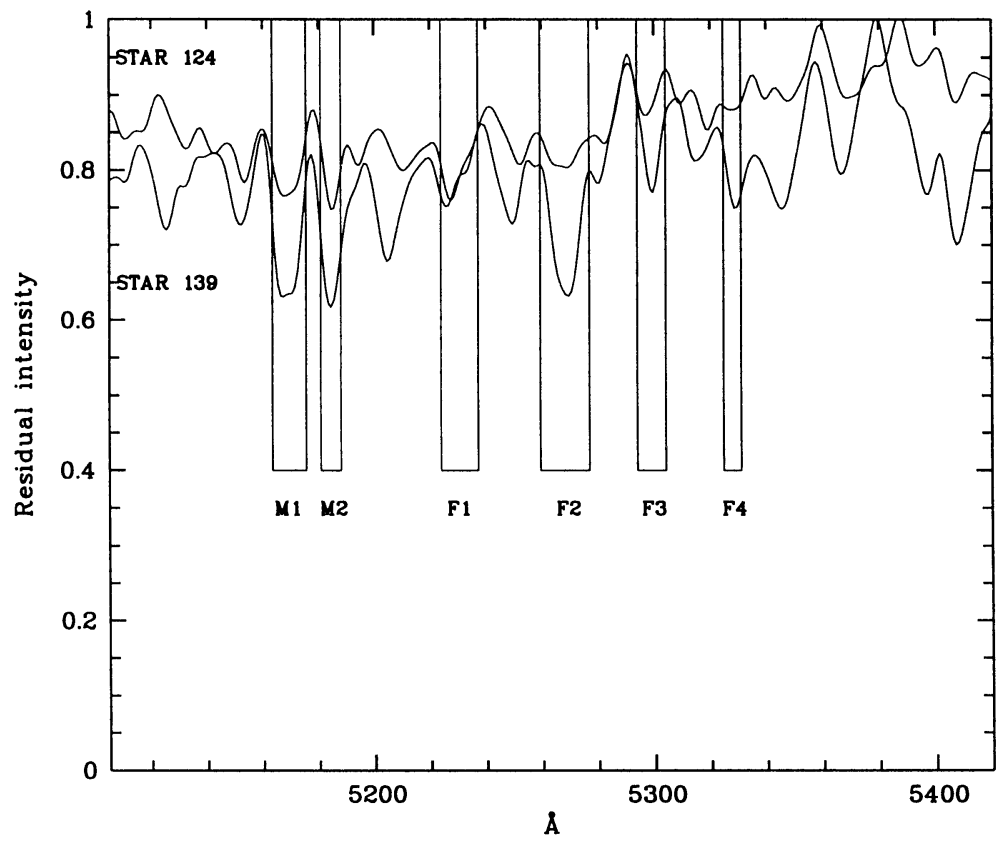

Figure 2. Comparison of the spectra of stars 124 and 139, which have the same $(V-I)_{0}$. The difference in line strengths has to be assigned to a different metallicity. The spectra have been smoothed with a Gaussian of width $300 \mathrm{~km} \mathrm{~s}^{-1}$ for display purposes. The solid boxes show the spectrophotometric indices used.

new synthetic spectrum using this scaling and $\mathrm{Mg}$ abundance and re-normalized the observed spectrum on this new synthetic spectrum and iterated the whole procedure until the change in metallicity was less than 0.2 dex. Typically three iterations were enough to meet this criterion. The procedure is quite CPU intensive and each iteration takes about three hours on the DEC Alpha of the OAT.

The effectiveness of the procedure was tested on the solar spectrum using both the Kurucz et al. (1984) solar atlas, degraded to the resolution of our grism spectra and twilight spectra. We were able to reproduce the solar $(\mathrm{Fe} / \mathrm{H})$ and $(\mathrm{Mg} / \mathrm{Fe})$ to within $0.1 \mathrm{dex}$. The values determined from the solar atlas for each index were used as zero point, so as to make our analysis strictly differential with respect to the Sun.

Three matters are of main concern in assessing the results obtained in this way: 1) the reliability of the line list we are using. The good results obtained for the Sun do not guarantee the completeness and correctness of the list, since our program stars are about $1000 \mathrm{~K}$ cooler and of much lower gravity. We shall try to quantify this issue in the future by analyzing the spectrum of a cool giant; 2) the placement of the continuum. The EMMI slitlets have ragged edges which do not allow a flux calibration, this, in conjunction with the low $\mathrm{S} / \mathrm{N}$ of our 
Table 2. Results

\begin{tabular}{ccccccc}
\hline$\#$ & $V_{0}$ & $\begin{array}{c}v_{r} \\
\mathrm{~km} \mathrm{~s}^{-1}\end{array}$ & $\begin{array}{c}T_{\text {eff }} \\
\mathrm{K}\end{array}$ & $\log \mathrm{g}$ & {$[\mathrm{Fe} / \mathrm{H}]$} & {$[\mathrm{Mg} / \mathrm{Fe}]$} \\
\hline 105 & 17.55 & 112 & 5041 & 2.59 & -0.5 & +0.1 \\
115 & 17.84 & 144 & 4953 & 2.36 & -1.0 & +1.2 \\
124 & 17.00 & 114 & 4891 & 2.21 & -0.6 & +0.3 \\
128 & 17.40 & 145 & 4778 & 1.93 & +0.7 & -0.5 \\
139 & 17.78 & 147 & 4891 & 2.21 & -0.1 & +0.4 \\
141 & 17.45 & 161 & 4977 & 2.42 & -0.6 & +0.4 \\
142 & 17.55 & 149 & 5118 & 2.81 & -0.7 & +0.2 \\
201 & 17.54 & 135 & 5003 & 2.49 & +0.1 & -0.0 \\
\hline
\end{tabular}

spectra, may induce an error in the continuum which is difficult to asses; 3) the microturbulent velocity has to be assumed, the results presented here are obtained for $\xi=2 \mathrm{~km} \mathrm{~s}^{-1}$. Clearly at this resolution we have no handle on microturbulence. What we shall do in a forthcoming paper is to quantify the effect of microturbulence on abundances. While these factors may affect our analysis in a systematic way (i.e. we may have a zero point uncertainty in the derived metallicities) they will not affect the relative abundances of the analyzed stars since the effective temperatures and gravities are quite similar.

\section{Discussion}

The results for eight of the 23 candidate members of Sgr $\mathrm{dSph}$ are presented in Table 2. Two facts stand out: 1) we do not find any star as metal-poor as $[\mathrm{Fe} / \mathrm{H}]=-1.5$, as was suggested by the photometry ; 2) three out of eight stars are metal-rich. Our metallicity scale may still suffer from a zero-point uncertainty which could make the most metal-poor stars we observe close to -1.5 , it is, however, interesting to note that a mismatch between photometric and spectroscopic metallicities in the same sense as the one we have here has been found by Francois et al. (1997) for the young halo globular cluster Ruprecht 106. Investigation of abundances of the whole available sample might allow to establish if we are in the presence of some systematic effect in abundance determinations or if what we see is merely a selection effect.

The presence of the metal-rich stars is disturbing, although they may in fact just be Bulge interlopers, three out of eight seems a rather high percentage. Furthermore, the metal-rich stars do not show signs of alpha-element enhancement such as are displayed by the Bulge $\mathrm{K}$ giants analyzed by McWilliam \& Rich (1994), but are more like the SMR stars in the solar neighbourhood. An independent investigation of metallicities of $\mathrm{Sgr} \mathrm{dSph}$ giants has been carried out by Smecker-Hane, McWilliam \& Ibata (1998) based on high-resolution spectra obtained at the Keck telescope, their results indicate a metallicity $\sim-1.5$ for the metal-poor stars, lower than our lowest metallicity stars, however, two out of seven stars they have analyzed are metal-rich and alpha-elements $\mathrm{O}$ and $\mathrm{Ca}$ are underabundant with respect to solar, in keeping with our results.

Probably the best way to decide whether these metal-rich stars belong to the Bulge or to Sagittarius is to determine the proper motions of the stars in their field. Bulge stars should show larger proper motions while Sagittarius 
stars should show a small and coherent proper motion. Such an investigation is within the capabilities of a diffraction limited instrument, such as HST, over a time baseline of just few years. At the distance of $8.5 \mathrm{kpc}$ a star with a transverse motion of $100 \mathrm{~km} \mathrm{~s}^{-1}$ would show a proper motion of $2.48 \mathrm{mas} \mathrm{yr}^{-1}$.

The future for abundance determinations for Sagittarius lies in the use of $8 \mathrm{~m}$ class telescopes. The $8.2 \mathrm{~m}$ VLT with the FORS1 instrument will allow to obtain spectra of these stars with $\mathrm{S} / \mathrm{N} \sim 100$, albeit with a slightly lower resolution. Moreover the slitlets of FORS1 in multi-object mode are sharp and will allow flux calibration of the spectra. Our technique of abundance estimation applied to such spectra should then be capable of producing abundances accurate to the 0.3 dex level.

Acknowledgements. We wish to thank F. Castelli for providing the theoretical colours and constant support in using the model-atmosphere and spectrum synthesis codes and A. Chieffi for providing the isochrones used in this work. Part of this work was done while P.B. was at ESO-Garching as a visiting scientist.

\section{References}

Alonso, A., Arribas, S., Martinez-Roger, C. 1996, A\&AS, 117, 227

Francois, P., Danziger, J.I., Buonanno, R., Perrin, N.M. 1997, A\&A, 327, 121

Ibata, R.A., Gilmore, G., Irwin, M.J. 1994, Nature, 370, 194

Ibata, R.A., Gilmore, G., Irwin, M.J. 1995, MNRAS, 277, 781

Ibata, R.A., Wyse, R.F.G., Gilmore, G., Irwin, M.J., Suntzeff, N.B. 1997, AJ, 113,634

Kurucz, R.L. 1993, CD-ROM No. 13, 18

Kurucz, R.L., Furenlid, I., Brault, J., Testerman, L. 1984, Solar Flux Atlas from 296 to $1300 \mathrm{~nm}$, Nat. Sol. Obs., Atlas No. 1

Marconi, G., Buonanno, R., Castellani, M., Iannicola, P., Molaro, P., Pasquini, L., Pulone, L. 1998, A\&A, 330, 453

McWilliam, A., Rich, R.M. 1994, ApJS, 91, 749

Smecker-Hane, T., McWilliam, A., Ibata, R.A. 1998, AAS Meeting, 192, 6613

Straniero, O., Chieffi, A., Limongi, M. 1997, ApJ, 490, 425

\section{Discussion}

Gulati: What are the models used as an input to synthesize spectra?

Marconi: Castelli, Gratton \& Kurucz. No overshooting input model of atmospheres computed with Atlas 9 code 2-enhanced ODFs, no-overshooting for the treatment of convection in Atlas.

Lequeux: Did you find carbon stars amongst your Sagittarius dwarf candidates? 
Marconi: No, because our sample was limited to $V-I \sim 1.3$ to avoid problems with model atmospheres.

Cannon: What exposure time did you use for each star?

Marconi: Several 20/30 minutes spectra up to 140 minutes. 